\title{
Dilated LMI characterization for the robust finite time control of discrete-time uncertain linear systems
}

\author{
Damiano Rotondo ${ }^{a}$, Fatiha Nejjari ${ }^{a}, V$ icenç Puig a,b \\ ${ }^{\mathrm{a}}$ Research Center for Supervision, Safety and Automatic Control (CS2AC), Universitat Politècnica de Catalunya (UPC), \\ Rambla de Sant Nebridi, 10, 08222 Terrassa, Spain. Tel: +34 937398973 \\ ${ }^{\mathrm{b}}$ Institut de Robotica i Informatica Industrial (IRI), UPC-CSIC Carrer de Llorens i Artigas, 4-6, 08028 Barcelona, Spain
}

\begin{abstract}
This paper provides new dilated linear matrix inequalities (LMIs) characterizations for the finite time boundedness (FTB) and the finite time stability (FTS) analysis of discrete-time uncertain linear systems. The dilated LMIs are later used to design a robust controller for the finite time control of discrete-time uncertain linear systems. The relevant feature of the proposed approach is the decoupling between the Lyapunov and the system matrices, that allows considering a parameter-dependent Lyapunov function. In this way, the conservativeness with respect to previous results is decreased. Numerical examples are used to illustrate the results.
\end{abstract}

Key words: Discrete-time systems; Finite time control; Linear matrix inequalities; Parameter-dependent Lyapunov functions, Robust control, Uncertain linear systems

\section{Introduction}

The idea of finite time stability (FTS) allows to analyze or design control systems that present some constraints on the state response without being necessarily stable. In fact, when the behavior of the system over a fixed time interval is of interest, a system could be defined stable when, given some initial conditions, the state remains within some desired bounds in such time interval, and unstable when it does not (Amato et al., 2001; Amato and Ariola, 2005; Amato et al., 2010b). Another concept which is strongly related to FTS is finite time boundedness (FTB), which takes into account norm bounded disturbances affecting the system (Amato et al., 2001). FTB implies FTS, but the converse is not true.

These concepts have been developed considerably in the last decade (Amato et al., 2014). In particular, the development of results based on differential/difference linear matrix inequalities (DLMIs) has attracted a lot of interest, mainly due to the possibility of providing necessary and sufficient conditions for the FTS/FTB of linear systems (Ichihara and Katayama, 2009; Amato et al., 2010a,b). In these works, it has been shown that the FTS of a time-varying linear system is guaranteed if and only if either a certain inequality involving the state transition matrix is satisfied, or a symmetric matrix function solving a certain Lyapunov differential/difference inequality exists. However, due to computational complexity issues, simpler sufficient (not necessary) conditions for FTS are used to address the problem of designing controllers guaranteeing some finite-time performance.

The problem of designing a robust finite time control law for uncertain systems has been solved only in the continuous-time case (Amato et al., 2001, 2011), and it seems that the discrete-time case represents still an open problem. In this paper, using the dilation technique suggested by de Oliveira et al. (1999), we provide new dilated linear matrix inequalities (LMIs) characterizations for the FTB and the FTS analysis. The dilated LMIs are used to

Email address: damiano.rotondo@yahoo.it (Damiano Rotondo). 
design a controller for the robust finite time control of discrete-time uncertain linear systems. The relevant feature of the proposed approach is the decoupling between the Lyapunov and the system matrices, that allows considering a Lyapunov function that depends on the parametric uncertainty. In this way, the conservativeness with respect to previous results, e.g. Amato et al. (2010b), is decreased. It is worth highlighting that in Amato et al. (2010b), time-varying linear systems without uncertainty are considered, in which case a time-varying Lyapunov matrix allows to obtain less conservative results than the ones obtained using a constant Lyapunov matrix. However, in the present work, the considered systems are subject to parametric uncertainty too, in which case using a Lyapunov function that associates a different Lyapunov matrix to each possible value of the unknown parametric uncertainty seems to be the most effective choice for reducing conservativeness with respect to parameter-independent Lyapunov functions.

\section{Preliminaries}

Let us recall the definitions of FTS and FTB in the case of discrete-time LTV systems (Amato et al., 2001; Amato and Ariola, 2005)

Definition 1 The discrete-time LTV system

$$
x(k+1)=A(k) x(k)
$$

is said to be finite time stable (FTS) with respect to $\left(c_{1}, c_{2}, N, R\right)$, with $c_{2}>c_{1}>0, N>0$ and $R>0$, if

$$
x(0)^{T} R x(0) \leq c_{1} \Rightarrow x(k)^{T} R x(k)<c_{2} \quad \forall k \in\{1, \ldots, N\}
$$

Definition 2 The discrete-time LTV system

$$
\left\{\begin{array}{l}
x(k+1)=A(k) x(k)+G(k) w(k) \\
w(k+1)=F(k) w(k)
\end{array}\right.
$$

is said to be finite time bounded (FTB) with respect to $\left(c_{1}, c_{2}, N, R, d\right)$, with $c_{2}>c_{1}>0, N>0, R>0$, and $d>0$ if

$$
\left\{\begin{array}{l}
x(0)^{T} R x(0) \leq c_{1} \\
w(0)^{T} w(0) \leq d
\end{array} \Rightarrow x(k)^{T} R x(k)<c_{2} \quad \forall k \in\{1, \ldots, N\}\right.
$$

Notice that FTS can be recovered as a special case of FTB when $w=0$. Finally, the following lemmas provide sufficient conditions for the FTB and the FTS of discrete-time LTV systems.

Lemma 1 Given $\rho \geq 1$, the discrete-time LTV system (3) is FTB with respect to $\left(c_{1}, c_{2}, N, R, d\right)$ if there exist positive scalars $\lambda_{1}, \lambda_{2}$ and two positive definite matrix-valued functions $Q_{1}(\cdot): k \in\{0,1, \ldots, N\} \mapsto Q_{1}(k) \in \mathbb{S}^{n_{x} \times n_{x}}$ and $P_{2}(\cdot): k \in\{0,1, \ldots, N\} \mapsto P_{2}(k) \in \mathbb{S}^{n_{w} \times n_{w}}$ such that

$$
\begin{aligned}
& \left(\begin{array}{cccc}
-\rho Q_{1}(k) & Q_{1}(k) A(k)^{T} & O & O \\
A(k) Q_{1}(k) & -Q_{1}(k+1) & G(k) & O \\
O & G(k)^{T} & -\rho P_{2}(k) & F(k)^{T} P_{2}(k+1) \\
O & O & P_{2}(k+1) F(k) & -P_{2}(k+1)
\end{array}\right)<0 \quad \forall k \in\{0,1, \ldots, N-1\} \\
& Q_{1}(0)>\lambda_{1} R^{-1} \\
& Q_{1}(k)<R^{-1} \quad \forall k \in\{1, \ldots, N\} \\
& P_{2}(0)<\lambda_{2} I \\
& \left(\begin{array}{cc}
\frac{c_{2}}{\rho^{N}}-\lambda_{2} d & \sqrt{c_{1}} \\
\sqrt{c_{1}} & \lambda_{1}
\end{array}\right)>0
\end{aligned}
$$


PROOF. The proof is inspired by the one provided in (Amato and Ariola, 2005) for the case of discrete-time LTI systems, and is omitted here due to space limitation.

Notice that by considering $F(k)=G(k)=O$ and $d=0$, conditions for analyzing the FTS of discrete-time LTV systems can be obtained. The analysis of FTS, with respect to FTB, is less demanding from a computational point of view, due to the lower size of the matrix inequalities and the lower number of variables that should be found (FTS analysis involves finding a positive scalar $\lambda_{1}$ and a positive definite matrix-valued function $Q_{1}(\cdot)$, contrarily to FTB analysis which involves finding in addition $\lambda_{2}$ and $\left.P_{2}(\cdot)\right)$. It can be shown that the sufficient condition for FTS of discrete-time LTV systems provided by Amato et al. (2010b) are a particular case obtained when $\rho=1$ and $c_{1}=1$.

\section{Main results}

The following theorems provide new dilated LMIs for analyzing the finite time boundedness and the finite time stability properties of discrete-time LTV systems.

Theorem 1 Given $\rho \geq 1$, the discrete-time LTV system (3) is FTB with respect to $\left(c_{1}, c_{2}, N, R, d\right)$ if there exist positive scalars $\lambda_{1}$ and $\lambda_{2}$, two positive definite matrix-valued functions $Q_{1}(\cdot): k \in\{0,1, \ldots, N\} \mapsto Q_{1}(k) \in \mathbb{S}^{n_{x} \times n_{x}}$ and $P_{2}(\cdot): k \in\{0,1, \ldots, N\} \mapsto P_{2}(k) \in \mathbb{S}^{n_{w} \times n_{w}}$, and two matrix-valued functions $H_{1}(\cdot): k \in\{0,1, \ldots, N-1\} \mapsto$ $H_{1}(k) \in \mathbb{R}^{n_{x} \times n_{x}}$ and $H_{2}(\cdot): k \in\{1, \ldots, N\} \mapsto H_{2}(k) \in \mathbb{R}^{n_{w} \times n_{w}}$ such that (6)-(9) and

$$
\left(\begin{array}{cccc}
-\rho\left(H e\left\{H_{1}(k)\right\}-Q_{1}(k)\right) & H_{1}(k)^{T} A(k)^{T} & O & O \\
A(k) H_{1}(k) & -Q_{1}(k+1) & G(k) & O \\
O & G(k)^{T} & -\rho P_{2}(k) & F(k)^{T} H_{2}(k+1) \\
O & O & H_{2}(k+1)^{T} F(k) & P_{2}(k+1)-H e\left\{H_{2}(k+1)\right\}
\end{array}\right)<0
$$

$\forall k \in\{0,1, \ldots, N-1\}$ hold

PROOF. The proof is inspired by the results obtained in de Oliveira et al. (1999). We first show that (5) implies (10). In fact, if (5) holds, we can choose $H_{1}(k)=H_{1}(k)^{T}=Q_{1}(k)$ and $H_{2}(k+1)=H_{2}(k+1)^{T}=P_{2}(k+1)$ in $(10)$ and recover (5).

It remains to show that (10) implies (5). To do so, let us assume that (10) holds, and let us notice that, for a given $k$, it can be rewritten as

$$
\begin{gathered}
\left(\begin{array}{cccc}
\rho Q_{1}(k) & O & O & O \\
O & -Q_{1}(k+1) & G(k) & O \\
O & G(k)^{T} & -\rho P_{2}(k) & O \\
O & O & O & P_{2}(k+1)
\end{array}\right)+H e\left\{\left(\begin{array}{cc}
-\rho I & O \\
A(k) & O \\
O & F(k)^{T} \\
O & -I
\end{array}\right) \Xi(k)\left(\begin{array}{cccc}
I & O & O & O \\
O & O & O & I
\end{array}\right)\right\}<0 \\
\Xi(k)=\left(\begin{array}{cc}
H_{1}(k) & O \\
O & H_{2}(k+1)
\end{array}\right)
\end{gathered}
$$

such that the Elimination Lemma (Gahinet and Apkarian, 1994) can be applied, resulting in

$$
\left(\begin{array}{cc}
\rho A(k) Q_{1}(k) A(k)^{T}-\rho^{2} Q_{1}(k+1) & \rho G(k) \\
\rho G(k)^{T} & F(k)^{T} P_{2}(k+1) F(k)-\rho P_{2}(k)
\end{array}\right)<0
$$

which, using a congruence transformation with $\operatorname{diag}\left(\rho^{-1} I, I\right)$, and applying Schur complements, can be shown to be equivalent to (5), thus completing the proof. 
Theorem 2 Given $\rho \geq 1$, the discrete-time LTV system (1) is FTS with respect to $\left(c_{1}, c_{2}, N, R\right)$ if there exist $\lambda>0$, a positive definite matrix-valued function $Q(\cdot): k \in\{0,1, \ldots, N\} \mapsto Q(k) \in \mathbb{S}^{n_{x} \times n_{x}}$, and a matrix-valued function $H(\cdot): k \in\{0,1, \ldots, N-1\} \mapsto H(k) \in \mathbb{R}^{n_{x} \times n_{x}}$ such that

$$
\left(\begin{array}{cc}
-\rho\left(H(k)+H(k)^{T}-Q(k)\right) & H(k)^{T} A(k)^{T} \\
A(k) H(k) & -Q(k+1)
\end{array}\right) \prec 0
$$

$\forall k \in\{0,1, \ldots, N-1\}$ and

$$
\begin{gathered}
Q(0)>\lambda R^{-1} \\
Q(k)<R^{-1} \quad \forall k \in\{1, \ldots, N\}
\end{gathered}
$$

hold.

PROOF. It is a direct consequence of Theorem 1 , when $F(k)=G(k)=O$ and $d=0$.

The new conditions introduced in Theorems 1 and 2 introduce the new variables $H_{1}(k), H_{2}(k)$ and $H(k)$ in order to achieve the separation of the Lyapunov matrices $Q_{1}(k), Q_{2}(k)$ and $Q(k)$ from the matrices $A(k)$ and $F(k)$. The advantage of doing so is that the new conditions allow to write new robust finite time boundedness (RFTB) and robust finite time stability (RFTS) conditions that are less conservative than the ones derived directly from Lemma 1.

To this aim, let us consider an uncertain LTV system, represented by

$$
\begin{gathered}
x(k+1)=\tilde{A}(k) x(k) \\
\left\{\begin{array}{l}
x(k+1)=\tilde{A}(k) x(k)+\tilde{G}(k) w(k) \\
w(k+1)=\tilde{F}(k) w(k)
\end{array}\right.
\end{gathered}
$$

with

$$
\begin{gathered}
\tilde{A}(k)=\sum_{i=1}^{N_{A}} \alpha_{i} A_{i}(k), \alpha_{i} \geq 0, \sum_{i=1}^{N_{A}} \alpha_{i}=1 \\
\tilde{F}(k)=\sum_{j=1}^{N_{F}} \phi_{j} F_{j}(k), \phi_{j} \geq 0, \sum_{j=1}^{N_{F}} \phi_{j}=1 \\
\tilde{G}(k)=\sum_{l=1}^{N_{G}} \gamma_{l} G_{l}(k), \gamma_{l} \geq 0, \sum_{l=1}^{N_{G}} \gamma_{l}=1
\end{gathered}
$$

Notice that the uncertainty representation in (19)-(21) is quite general, and can be regarded as structured uncertainty. Then, the RFTB and the RFTS are defined as follows.

Definition 3 The uncertain LTV system (17) is said to be RFTS with respect to $\left(c_{1}, c_{2}, N, R\right)$, with $c_{2}>c_{1}>0$, $N>0$ and $R>0$, if and only if it is FTS for all $\tilde{A}(k)$.

Definition 4 The uncertain LTV system (18) is said to be RFTB with respect to $\left(c_{1}, c_{2}, N, R, d\right)$, with $c_{2}>c_{1}>0$, $N>0, R>0$ and $d>0$, if and only if it is FTB for all $\tilde{A}(k), \tilde{F}(k)$ and $\tilde{G}(k)$.

Then, the following theorems provide sufficient conditions for the RFTB and the RFTS of the uncertain LTV systems (18) and (17), respectively.

Theorem 3 Given $\rho \geq 1$, the uncertain discrete-time LTV system (18) with uncertainty domain given by (19)-(21) is RFTB with respect to $\left(c_{1}, c_{2}, N, R, d\right)$ if there exist positive scalars $\lambda_{1, i j l}$ and $\lambda_{2, i j l}$, positive definite matrix-valued functions $Q_{1, i j l}(\cdot): k \in\{0,1, \ldots, N\} \mapsto Q_{1, i j l}(k) \in \mathbb{S}^{n_{x} \times n_{x}}$ and $P_{2, i j l}(\cdot): k \in\{0,1, \ldots, N\} \mapsto P_{2, i j l}(k) \in \mathbb{S}^{n_{w} \times n_{w}}$ and two matrix-valued functions $H_{1}(\cdot): k \in\{0,1, \ldots, N-1\} \mapsto H_{1}(k) \in \mathbb{R}^{n_{x} \times n_{x}}$ and $H_{2}(\cdot): k \in\{1, \ldots, N\} \mapsto H_{2}(k) \in \mathbb{R}^{n_{w} \times n_{w}}$ such that 


$$
\left(\begin{array}{cccc}
\rho\left(Q_{1, i j l}(k)-H e\left\{H_{1}(k)\right\}\right) & H_{1}(k) A_{i}(k)^{T} & O & O \\
A_{i}(k) H_{1}(k) & -Q_{1, i j l}(k+1) & G_{l}(k) & O \\
O & G_{l}(k)^{T} & -\rho P_{2, i j l}(k) & F_{j}(k)^{T} H_{2}(k+1) \\
O & O & H_{2}(k+1)^{T} F_{j}(k) & P_{2, i j l}(k+1)-H e\left\{H_{2}(k+1)\right\}
\end{array}\right)<0
$$

$\forall k \in\{0,1, \ldots, N-1\}$ and

$$
\begin{gathered}
Q_{1, i j l}(0)>\lambda_{1, i j l} R^{-1} \\
Q_{1, i j l}(k)<R^{-1} \quad \forall k \in\{1, \ldots, N\} \\
P_{2, i j l}(0)<\lambda_{2, i j l} I \\
\left(\begin{array}{cc}
\frac{c_{2}}{\rho^{N}}-\lambda_{2, i j l} d & \sqrt{c_{1}} \\
\sqrt{c_{1}} & \lambda_{1, i j l}
\end{array}\right)>0
\end{gathered}
$$

hold for all $i=1, \ldots, N_{A}, j=1, \ldots, N_{F}$ and $l=1, \ldots, N_{G}$.

PROOF. Due to a basic property of matrices (Horn and Johnson, 1990), any linear combination of positive (negative) definite matrices with non-negative coefficients, of which at least one different from zero, is positive (negative) definite. Hence, using the coefficients $\alpha_{i}, \phi_{j}$ and $\gamma_{l}$ defined in (19)-(21), it follows from (22)-(26) that (10) and (6)-(9) hold for any $\tilde{A}(k), \tilde{F}(k)$ and $\tilde{G}(k)$. It follows that Theorem 1 holds for any $\tilde{A}(k), \tilde{F}(k)$ and $\tilde{G}(k)$, i.e. the uncertain LTV system (18) is RFTB with respect to $\left(c_{1}, c_{2}, N, R, d\right)$. This completes the proof.

Theorem 4 Given $\rho \geq 1$, the uncertain discrete-time LTV system (17) with uncertainty domain (19) is RFTS with respect to $\left(c_{1}, c_{2}, N, R\right)$ if there exist positive scalars $\lambda_{i}$, positive definite matrix-valued functions $Q_{i}(\cdot): k \epsilon$ $\{0,1, \ldots, N\} \mapsto Q_{i}(k) \in \mathbb{S}^{n_{x} \times n_{x}}$ and a matrix-valued function $H(\cdot): k \in\{0,1, \ldots, N-1\} \mapsto H(k) \in \mathbb{R}^{n_{x} \times n_{x}}$ such that

$$
\left(\begin{array}{cc}
\rho\left(Q_{i}(k)-H e\{H(k)\}\right) & H(k)^{T} A_{i}(k)^{T} \\
A_{i}(k) H(k) & -Q_{i}(k+1)
\end{array}\right)<0
$$

$\forall k \in\{0,1, \ldots, N-1\}$ and

$$
\begin{gathered}
Q_{i}(0)>\lambda_{i} R^{-1} \\
Q_{i}(k)<R^{-1} \quad \forall k \in\{1, \ldots, N\} \\
\left(\begin{array}{cc}
\frac{c_{2}}{\rho^{N}} & \sqrt{c_{1}} \\
\sqrt{c_{1}} & \lambda_{i}
\end{array}\right)>0
\end{gathered}
$$

hold for all $i=1, \ldots, N_{A}$.

PROOF. The proof is similar in the reasoning to the one of Theorem 3, thus it is omitted.

It is worth noticing that, contrarily to Theorems 1 and 2 , that do not use a parameter-dependent Lyapunov function (the matrix-valued functions $Q_{1}(\cdot)$ and $P_{2}(\cdot)$ do not depend on the uncertainty), in Theorems 3 and 4 the parameterdependent Lyapunov functions are used.

The conditions presented in Theorem 3 and 4 can be easily extended to solve the problem of the finite time control of uncertain LTV systems. Due to space limitation, only the case of RFTB control will be discussed. To this aim, let us consider uncertain LTV systems of the form

$$
\left\{\begin{array}{l}
x(k+1)=\tilde{A}(k) x(k)+\tilde{B}(k) u(k)+\tilde{G}(k) w(k) \\
w(k+1)=\tilde{F}(k) w(k)
\end{array}\right.
$$


with $\tilde{A}(k), \tilde{F}(k)$ and $\tilde{G}(k)$ defined as in (19)-(21), and

$$
\tilde{B}(k)=\sum_{h=1}^{N_{B}} \beta_{h} B_{h}(k), \beta_{h} \geq 0, \sum_{h=1}^{N_{B}} \beta_{h}=1
$$

and let us search the gain $K(k)$ for the state-feedback control law

$$
u(k)=K(k) x(k)
$$

such that the resulting closed-loop system

$$
\left\{\begin{array}{l}
x(k+1)=(\tilde{A}(k)+\tilde{B}(k) K(k)) x(k)+\tilde{G}(k) w(k) \\
w(k+1)=\tilde{F}(k) w(k)
\end{array}\right.
$$

is RFTB with respect to $\left(c_{1}, c_{2}, N, R, d\right)$. The following corollary provides a solution to this problem.

Corollary 1 Given $\rho \geq 1$, the uncertain discrete-time LTV system (31) with uncertainty domain given by (19)-(21) and (32), under control law (33), is RFTB with respect to $\left(c_{1}, c_{2}, N, R, d\right)$ if there exist positive scalars $\lambda_{1, \text { hijl }}$, $\lambda_{2, h i j l}$, positive definite matrix-valued functions $Q_{1, i h j l}(\cdot): k \in\{0,1, \ldots, N\} \mapsto Q_{1, h i j l}(k) \in \mathbb{S}^{n_{x} \times n_{x}}$ and $P_{2, h i j l}(\cdot)$ : $k \in\{0,1, \ldots, N\} \mapsto P_{2, h i j l} \in \mathbb{S}^{n_{w} \times n_{w}}$, and matrix-valued functions $H_{1}(\cdot): k \in\{0,1, \ldots, N-1\} \mapsto H_{1}(k) \in \mathbb{R}^{n_{x} \times n_{x}}$, $H_{2}(\cdot): k \in\{1,2, \ldots, N\} \mapsto H_{2}(k) \in \mathbb{R}^{n_{w} \times n_{w}}$ and $\Gamma(\cdot): k \in\{0,1, \ldots, N-1\} \mapsto \Gamma(k) \in \mathbb{R}^{n_{u} \times n_{x}}$ such that

$$
\left(\begin{array}{cccc}
\rho Q_{1, h i j l}(k) & O & O & O \\
O & -Q_{1, h i j l}(k+1) & G_{l}(k) & O \\
O & G_{l}(k)^{T} & -\rho P_{2, h i j l}(k) & O \\
O & O & O & P_{2, h i j l}(k+1)
\end{array}\right)+H e\left\{\left(\left(\begin{array}{clll}
-\rho H_{1}(k) & O & O \\
\Omega_{i h}(k) & O & O & O \\
O & O & O & F(k)^{T} H_{2}(k+1) \\
O & O & O & -H_{2}(k+1)
\end{array}\right)\right\}<0\right.
$$

$\forall k \in\{0,1, \ldots, N-1\}$ and

$$
\begin{gathered}
\Omega_{i h}(k)=A_{i}(k) H_{1}(k)+B_{h}(k) \Gamma(k) \\
Q_{1, h i j l}(0)>\lambda_{1, h i j l} R^{-1} \\
Q_{1, h i j l}(k)<R^{-1} \quad \forall k \in\{1, \ldots, N\} \\
P_{2, h i j l}(0)<\lambda_{2, h i j l} I \\
\left(\begin{array}{cc}
\frac{c_{2}}{\rho^{N}}-\lambda_{2, h i j l} d & \sqrt{c_{1}} \\
\sqrt{c_{1}} & \lambda_{1, h i j l}
\end{array}\right)>0
\end{gathered}
$$

hold for all $h=1, \ldots, N_{B}, i=1, \ldots, N_{A}, j=1, \ldots, N_{F}$ and $l=1, \ldots, N_{G}$ and if the controller gain is chosen as $K(k)=\Gamma(k) H_{1}(k)^{-1}$.

PROOF. This corollary follows from Theorem 3, taking into account that the closed-loop system is described by (34), and through the change of variable $\Gamma(k)=K(k) H_{1}(k)$.

From a computational point of view, once a value for $\rho$ is fixed, the feasibility of the conditions stated in Theorems 3-4 and Corollary 1 are linear matrix inequalities (LMIs) feasibility problems.

\section{Illustrative examples}

In the following, the DLMI-based FTS analysis results provided by Amato et al. (2013) are compared with the proposed RFTS analysis condition obtained through the process of dilation (see Theorem 4). To this aim, let us consider 100 random realizations of the uncertain LTV system (17), with the matrix $\tilde{A}(k) \in \mathbb{R}^{3 \times 3}$ satisfying (19), and let us find the minimum value of $c_{2}$ that guarantees the feasibility of the analysis condition when $c_{1}=1, N=4$, $R=I$ and $\rho=1$. The comparison between the conditions presented by Amato et al. (2013) and the one given by 

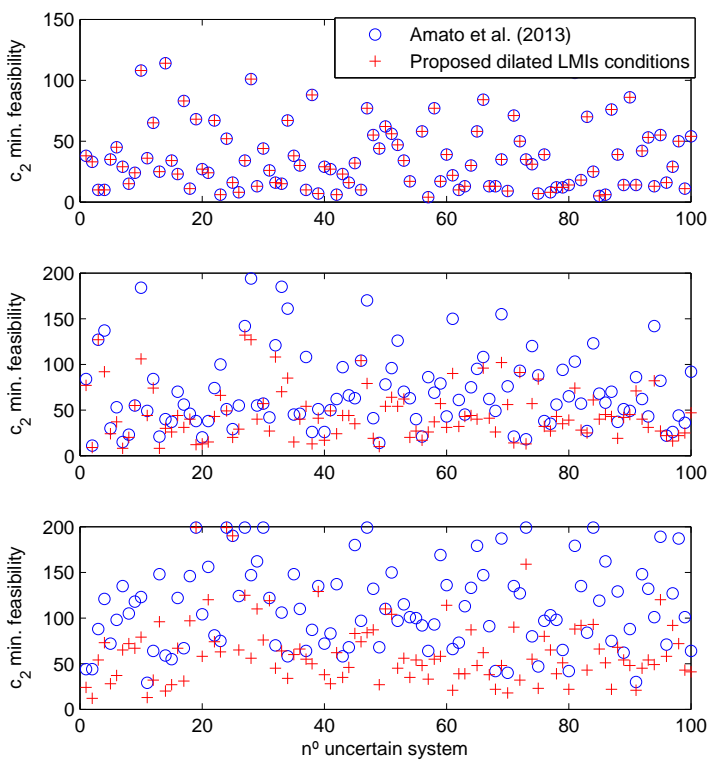

Fig. 1. Minimum value of $c_{2}$ that provides feasibility of the analysis conditions for $N_{A}=1, N_{A}=2$ and $N_{A}=3$. Comparison between the conditions from Amato et al. (2013) and the proposed dilated LMIs conditions (Theorem 4).

Theorem 4 will be performed in the cases $N_{A}=1, N_{A}=2$ and $N_{A}=3$ (notice that the first case corresponds to the absence of uncertainty). It is worth highlighting that in the cases $N_{A}=2$ and $N_{A}=3$, the conditions proposed by Amato et al. (2013) are slightly modified in order to take into account the presence of uncertainty, by assuming that they hold for every value of $\tilde{A}(k)$.

In Fig. 1, it can be seen that, when $N_{A}=1$ (no uncertainty), there are no differences between the results obtained with the analysis conditions obtained from Amato et al. (2013) and the RFTS analysis by dilated LMIs proposed in Theorem 4. In fact, the case $N_{A}=1$ corresponds to Theorem 2, in which case an exact equivalence between the non-dilated LMIs and the dilated LMIs holds (see proof of Theorem 1). On the other hand, when $N_{A}>1$, the proposed dilated LMIs exhibit less conservativeness (in the sense of obtaining feasible LMIs for a smaller value of $c_{2}$ ) than the conditions obtained from Amato et al. (2013).

Finally, let us consider an uncertain LTV system as in (31), with $\tilde{A}(k), \tilde{B}(k), \tilde{F}(k), \tilde{G}(k)$ defined as in (19)-(21) and (32), with $N_{A}=N_{B}=N_{F}=N_{G}=3$ and with matrices $A_{i}(k) \in \mathbb{R}^{3 \times 3}, B_{h}(k) \in \mathbb{R}^{3 \times 1}, F_{j}(k) \in \mathbb{R}^{1 \times 1}, G_{l}(k) \in \mathbb{R}^{3 \times 1}$, $i=1,2,3, h=1,2,3, j=1,2,3, l=1,2,3$ and $k=0,1,2,3$, and let us consider the problem of finding a state-feedback control law (33) such that (34) is RFTB with respect to $\left(1, c_{2}, 4, I, 1\right)$ (see Definition 2 for the meaning of each value in this quintuplet). Using Corollary 1 , a minimum value $c_{2}=59$ for which the LMIs exhibit feasibility is obtained. A comparison of the open loop and the closed loop trajectories, obtained for 100 random realizations of the uncertain LTV system, with random initial conditions satisfying $x(0)^{T} x(0)=1$ and $w(0)^{T} w(0)=1$, is shown in Fig. 2 . It can be seen that in the open loop case $x(k)^{T} x(k)>59$ for some simulations (red lines). Hence, the open loop system is not RFTB with respect to $(1,59,4, I, 1)$. On the other hand, for the closed loop system $x(k)^{T} x(k)<59$ in all the simulations, thus demonstrating its RFTB with respect to $(1,59,4, I, 1)$.

\section{Conclusions}

New robust finite time boundedness and robust finite time stability conditions for uncertain discrete-time LTV systems have been provided. These conditions, which have the relevant feature of decoupling the Lyapunov matrices from the systems ones, have been obtained through a process of dilation, starting from existing results about finite time control. Using these conditions, time-varying parameter-dependent Lyapunov matrices can be considered, allowing to overcome the conservativeness with respect to time-varying not parameter-independent Lyapunov matrices. Finally, the problem of robust finite time control has been solved. Simulation results have demonstrated the reduced 


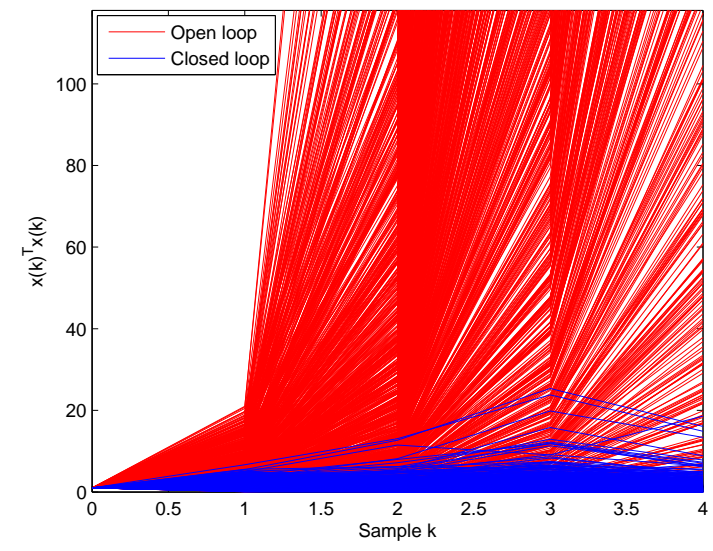

Fig. 2. Comparison between open loop and closed loop trajectories with a controller designed to be RFTB with respect to $(1,59,4, I, 1)$ using Corollary 1.

conservativeness of the proposed conditions with respect to the existing results, as well as proved the effectiveness of the proposed conditions in achieving robust finite time control in presence of uncertainties.

\section{Acknowledgements}

This work has been funded by the Spanish MINECO through the projects CICYT ECOCIS (ref. DPI2013-48243C2-1-R) and CICYT HARCRICS (Ref. DPI2014-58104-R), by AGAUR through the contracts FI-DGR 2014 (ref. 2014FI_B1 00172) and FI-DGR 2015 (ref. 2015FI_B2 00171) and by the DGR of Generalitat de Catalunya (SAC group Ref. 2014/SGR/374).

\section{References}

F. Amato and M. Ariola. Finite-time control of discrete-time linear systems. IEEE Transactions on Automatic Control, 50(5):724-729, 2005.

F. Amato, M. Ariola, and P. Dorato. Finite-time control of linear systems subject to parametric uncertainties and disturbances. Automatica, 37(9):1459-1463, 2001.

F. Amato, M. Ariola, and C. Cosentino. Finite-time stability of linear time-varying systems: analysis and controller design. IEEE Transactions on Automatic Control, 55(4):1003-1008, 2010a.

F. Amato, M. Ariola, and C. Cosentino. Finite-time control of discrete-time linear systems: analysis and design conditions. Automatica, 46(5):919-924, 2010 b.

F. Amato, M. Ariola, and C. Cosentino. Robust finite-time stabilisation of uncertain linear systems. International Journal of Control, 84(12):2117-2127, 2011.

F. Amato, G. De Tommasi, and A. Pironti. Necessary and sufficient conditions for finite-time stability of impulsive dynamical linear systems. Automatica, 49:2546-2550, 2013.

F. Amato, R. Ambrosino, M. Ariola, C. Cosentino, and G. De Tommasi. Finite-time stability and control. Springer, London, UK, 2014.

M. C. de Oliveira, J. Bernussou, and J. C. Geromel. A new discrete-time robust stability condition. Systems and Control Letters, 37(4):261-265, 1999.

P. Gahinet and P. Apkarian. A linear matrix inequality approach to $\mathcal{H}_{\infty}$ control. International Journal of Robust and Nonlinear Control, 4(4):421-448, 1994.

R. A. Horn and C. R. Johnson. Matrix Analysis. Cambridge University Press, 1990. ISBN 978-0-521-38632-6.

H. Ichihara and H. Katayama. Necessary and sufficient conditions for finite-time boundedness of linear continuoustime systems. In Proceedings of the Joint 48th IEEE Conference on Decision and Control (CDC) and 28th Chinese Control Conference (CCC), pages 3214-3219, 2009. 